\title{
Residue after Decontamination
}

National Cancer Institute

\section{Source}

National Cancer Institute. Residue after Decontamination. NCI Thesaurus. Code C62850.

Problem associated with the decontamination process not adequately removing unwanted visible soil, foreign material, or org anism deposits. 